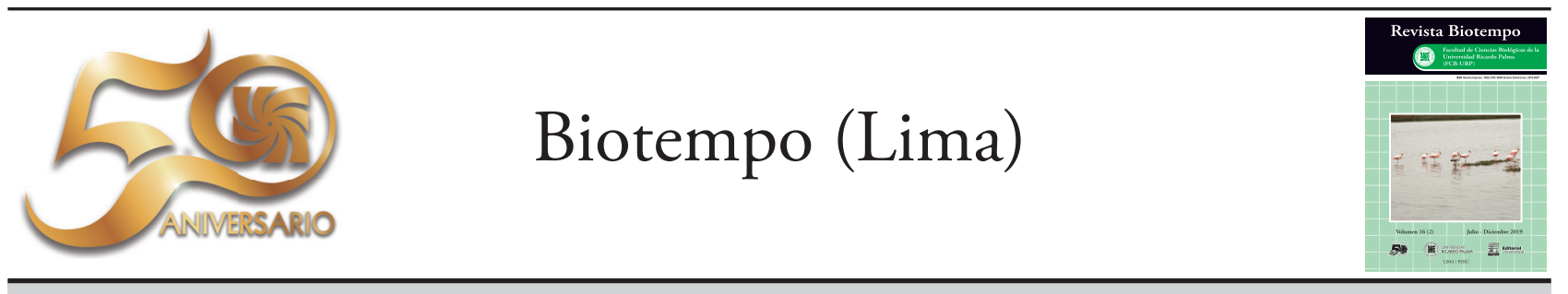

ORIGINAL ARTICLE / ARTÍCULO ORIGINAL

EFFECTS OF $\beta$-CARIOFYLENE OF PIPER NIGRUM EXTRACT IN THE SPERM HYPERACTIVATION AND IN THE FERTILIZING CAPACITY OF TETRAPYGUS NIGER (MOLINA, 1782) "BLACK SEA URCHIN"

\title{
EFECTOS DEL EXTRACTO DE $\beta$-CARIOFILENO DE PIPER NIGRUM EN LA HIPERACTIVACIÓN ESPERMÁTICA Y EN LA CAPACIDAD FECUNDANTE DE TETRAPYGUS NIGER (MOLINA, 1782) "ERIZO NEGRO DE MAR"
}

\section{Daniela Zapata $^{1}$; Joyce Wixsan ${ }^{1}$; Katherine Hinostroza ${ }^{1}$; Santiago Justo ${ }^{1}$; Hugo Gonzales-Figueroa ${ }^{1}$}

Laboratorio de Biotecnología Animal. Facultad de Ciencias Biológicas, Universidad Ricardo Palma, Lima, Perú.

2 Department of Biochemistry - Institute of Chemistry University of Sao Paulo, Brasil.

Author for correspondence: hgonzales@urp.edu.pe

\begin{abstract}
We analyzed hyperactivation and fertilizing capacity of the sperm of Tetrapygus niger "black sea urchin" in the presence of $\beta$-caryophyllene. $\beta$-caryophyllene obtained from extracts of Piper nigrum "black pepper" and synthetic $\beta$-caryophyllene donated by the Biosciences Institute of the University of Sao Paulo, Sao Paulo, Brazil, was used. Tetrapygus niger spermatozoa pretreated with the extracts of synthetic $\beta$-caryophyllene ( $\beta$-caryophyllene $A)$ and $\beta$-Caryophyllene from black pepper ( $\beta$-caryophyllene $B$ ) were used to study the effect of this molecule on sperm hyperactivity (mean velocity and percentages of progressive and continuous movement) and the fertilizing capacity (percentage of fertilized ovules). The average values of the sperm head area in the control and in the treatments with $\beta$ caryophyllene $A$ and $\beta$-caryophyllene B were: 33.8 ; 32.33 and $33.59 \mathrm{um}^{2}$, respectively, indicating the homogeneity in the sample and that this was not influenced by the sesquiterpene. In the presence of $\beta$-caryophyllene $A$ and $B$, the average speed observed was decreased by approximately 30 and $20 \mathrm{um} / \mathrm{s}$, respectively, with respect to the control. There are significant differences between the control and the treatments with $\beta$-caryophyllene $A$ and $B$, but not between the two treatments with $\beta$-caryophyllene. The progressive movement of the sperm was also affected. While all the sperm of the control group showed rapid progressivity; in the treatment with $\beta$-caryophyllene B only $40 \%$ presented this characteristic and in the treatment with $\beta$-caryophyllene A $100 \%$ were progressive slow. On the other hand, the $\beta$-caryophyllene B produced a slight decrease in fertilization (96.76\% of fertilized ovules) and in the presence of $\beta$-caryophyllene $A$, this was reduced to $79.50 \%$ with respect to the control group. A possible mechanism to explain the effects observed in this study would be the ability of $\beta$-caryophyllene to activate inhibitory $\mathrm{G}$ proteins as reported in other studies.
\end{abstract}

Keywords: $\beta$-caryophyllene - fertilizing capacity - sperm hyperactivation 


\section{RESUMEN}

En el presente trabajo se analizó la hiperactivación espermática y la capacidad fecundante del espermatozoide de Tetrapygus niger (Molina, 1782) "erizo negro de mar" en presencia de $\beta$-cariofileno. Se utilizó el sesquiterpeno $\beta$-cariofileno obtenido a partir de extracto de Piper nigrum L. "pimienta negra” y de $\beta$-cariofileno sintético donado por el Instituto de Biociencias de la Universidad de Sao Paulo (Sao Paulo, Brasil). Espermatozoides de Tetrapygus niger pretratados con los extractos de $\beta$-cariofileno sintético $(\beta$-cariofileno $A$ ) y $\beta$-cariofileno de pimienta negra ( $\beta$-cariofileno $B$ ) fueron utilizados para la hiperactividad espermática (velocidad media y porcentajes de movimiento progresivo y continuo) y la capacidad fecundante (porcentaje de óvulos fecundados). Los valores promedios del área de la cabeza del espermatozoide en el control y en los tratamientos con $\beta$-cariofileno A y $\beta$-cariofileno B fueron de: 33,8; 32,33 y 33,59 um², respectivamente, indicando la homogeneidad en la muestra y que esta no fue influenciada por el sesquiterpeno. En presencia de $\beta$-cariofileno A y B se observó la disminución de la velocidad promedio en aproximadamente 30 y $20 \mathrm{um} / \mathrm{s}$, respectivamente, con respecto al control; existiendo diferencias significativas entre el control y los tratamientos con $\beta$-cariofileno $\mathrm{A}$ y $\mathrm{B}$, pero no entre los dos tratamientos con $\beta$-cariofileno. El movimiento progresivo de los espermatozoides también fue afectado, mientras la totalidad de los espermatozoides del grupo control presentaron progresividad rápida, en el tratamiento con $\beta$-cariofileno B solo el $40 \%$ presentaron esta característica y en el tratamiento con $\beta$-cariofileno A el $100 \%$ fueron progresivos lento. Por otro lado, el $\beta$-cariofileno B produjo una ligera disminución de fecundación (96,76\% de óvulos fecundados) y en presencia de $\beta$-cariofileno $A$, esta se redujo a 79,50 \% con respecto al grupo control. Un posible mecanismo para explicar los efectos observados en este estudio sería la capacidad del $\beta$-cariofileno para activar proteínas $\mathrm{G}$ inhibitorias, como ha sido reportado en otros estudios.

Palabras clave: $\beta$ cariofileno - capacidad fecundante - hiperactivacion espermática

\section{INTRODUCCIÓN}

Los espermatozoides de erizo de mar son capaces de fusionarse con el óvulo después de una serie de modificaciones estructurales, funcionales y moleculares, denominadas capacitación y reacción del acrosoma (Bernabó et al., 2012). Además, adquieren motilidad solo cuando entran en contacto con el agua de mar (Neil \& Vacquier, 2004) y para aumentar la tasa de éxito de la fecundación, son atraídos por moléculas dispersadas por los óvulos que en su cubierta gelatinosa contiene péptidos activadores del espermatozoide (SAP) que se unen a receptores específicos y promueven la quimiotaxis. Cuando los espermatozoides se encuentran con el óvulo ocurre la reacción del acrosoma y la polimerización de la actina que conduce a la extensión del túbulo acrosomal que permitirá la fusion de las membranas del espermatozoide con la del óvulo (Kamei \& Gable, 2003).

El sistema endocannabinoide es una vía de señalización endógena que está ampliamente distribuída en los vertebrados e invertebrados, el cual participa en múltiples vías metabólicas regulando de forma versátil la fisiología celular. Este sistema está constituido por los receptores de cannabinoides, sus agonistas endógenos o endocannabinoides, las enzimas responsables de la síntesis y degradación de los mismos y las vías de señalización intracelular reguladas por los endocannabinoides, así como los sistemas de transporte (Lu \& Mackie, 2016). Los receptores cannabinoides $\mathrm{CB}_{1}$ y $\mathrm{CB}_{2}$ están acoplados a la proteína $\mathrm{G}$ y se expresan en mamíferos, aves, reptiles e invertebrados como equinodermos (McPartland et al., 2006).

Existen evidencias que en la membrana plasmática del espermatozoide de Strongylocentrotus purpuratus (Stimpson, 1857) "erizo de mar" se localiza un receptor cannabinoide que tiene como agonista una sustancia proveniente de la capa gelatinosa del huevo o cigote, que cuando es bloqueado por un cannabinoide psicoactivo de Cannabis sativa, reduce la capacidad de fecundación del espermatozoide (Chang et al., 1993). Asimismo, en estos espermatozoides en presencia de tetrahidrocannabinol (THC), cannabidiol (CBD) y cannabinol (CBN), agonistas de los receptores cannabinoides $\mathrm{CB}_{1}$ y $\mathrm{CB}_{2}$, se inhibe la reacción del acrosoma, impidiendo la fecundación (Schuel et al., 1991).

El $\beta$-cariofileno es un sesquiterpeno cannabinoide que actúa como agonista natural selectivo del receptor cannabinoide CB2 (Gertsch, 2008). Se encuentra en grandes cantidades en los aceites esenciales de muchas especias y plantas alimenticias, como Origanum vulgare L. “orégano" (Mockute et al., 2001), Cinnamomum 
spp. "canela” (Jayaprakasha et al., 2003) y P. nigrum L. " pimienta negra” (Orav et al., 2004) y es el mayor componente en C. sativa.

Se ha reportado que el receptor cannabinoide $\mathrm{CB}_{2}$ presente en la superficie de la membrana plasmática de los espermatozoides humanos regula la motilidad espermática (Agirregoitia et al., 2010).

En el presente trabajo se analiza la hiperactivación espermática y la capacidad fecundante del espermatozoide de T. niger "erizo negro de mar" en presencia del sesquiterpeno $\beta$-cariofileno, de extracto de pimienta negra y sintético.

\section{MATERIALES Y MÉTODOS}

\section{Material biológico}

\section{Obtención de $\beta$-cariofileno de Piper nigrum $y$ $\beta$-cariofileno sintético}

La obtención de $\beta$-cariofileno se realizó en el laboratorio de Química de la Facultad de Ciencias Biológicas (FCB) de la Universidad Ricardo Palma (URP), Lima, Perú. La extracción de $\beta$-cariofileno a partir de $P$. nigrum se realizó siguiendo la metodología descrita por Zhou et al. (2012) con algunas modificaciones. $P$. nigrum seca se maceró durante una semana en metanol. Posteriormente, se utilizaron dos etapas de cromatografia en capa fina para purificar el $\beta$-cariofileno. En la primera etapa se utilizó como fase móvil una mezcla de cloroformo:metanol 9:1, dejando correr la muestra hasta alcanzar $30 \mathrm{~mm}$; luego, en la segunda etapa se usó éter de petróleo:etil acetato 10:1, dejando correr la muestra hasta alcanzar $50 \mathrm{~mm}$. La presencia de $\beta$-cariofileno se confirmó utilizando revelador de vanilina en $\mathrm{H}_{2} \mathrm{SO}_{4} 1 \%$. El $\beta$-cariofileno fue recuperado de la silica gel por raspado y posteriormente extraído con éter etílico. Finalmente, el éter etílico se evaporó en baño maría y el extracto final obtenido fue utilizado para el ensayo biológico.

El $\beta$-cariofileno sintético fue donado por el Instituto de Biociencias de la Universidad de Sao Paulo (Sao Paulo, Brasil).

\section{Colecta de especímenes de T. niger "erizo negro de mar"}

Los especímenes de T. niger "erizo negro de mar" se recolectaron en las rocas ubicadas en la orilla del muelle de la playa Pescadores de Chorrillos (-12.164935, -77.029915), Lima, Perú fueron colocados en agua de mar a temperatura ambiente $\left(14-16^{\circ} \mathrm{C}\right)$ e inmediatamente transportados al Laboratorio de Biotecnología Animal de la FCB, URP.

\section{Obtención y preparación de los gametos}

Los especímenes colectados se mantuvieron en un recipiente provisto con un aireador y agua de mar, la cual fue renovada continuamente. Los gametos se obtuvieron por estimulación química, inyectando en la cavidad oral, de cada espécimen, $1 \mathrm{~mL}$ de una solución de $\mathrm{KCl}$ 0,15 M.

Para la preparación del control, se diluyó 100 uL de espermatozoides en $100 \mathrm{~mL}$ de agua de mar estéril y se homogeneizó lentamente. Para el tratamiento con $\beta$-cariofileno sintético ( $\beta$-cariofileno A) se adicionó $10 \mathrm{uL}$ de este a $100 \mathrm{~mL}$ de agua de mar estéril, asumiendo una pureza de $100 \%$ se obtendría una concentración final de $\beta$-cariofileno de $10 \mathrm{uM}$. Posteriormente se ańadió a la solución $100 \mathrm{uL}$ de espermatozoides y se homogeneizó lentamente. Se procedió del mismo modo para el tratamiento con extracto de $\beta$-cariofileno $(\beta$-cariofileno B). Los óvulos, previamente lavados, fueron diluidos en $100 \mathrm{~mL}$ de agua de mar estéril (Olaechea et al., 2006).

\section{Fecundación in vitro}

Para el ensayo de fecundación se agregó la dilución control de espermatozoides a la dilución de óvulos, se dejó reposar por $10 \mathrm{~min}$, se homogeneizó la dilución y se extrajo una gota para su observación en el microscopio óptico. Se cuantificaron los óvulos fecundados y los no fecundados en cada campo de 3 láminas preparadas. Se repitió el procedimiento para las diluciones de espermatozoides tratados con los extractos A y B de $\beta$-cariofileno (Olaechea et al., 2006).

\section{Hiperactivación espermática}

Para el análisis de la hiperactividad espermática se utilizaron también los 3 tratamientos de espermatozoides (control, tratamiento con $\beta$-cariofileno A y tratamiento con $\beta$-cariofileno $B$ ), colocando una gota de la dilución en una placa Petri para su posterior observación en un microscopio invertido conectado a una cámara. Se generaron videos e imágenes por cada tratamiento.

\section{Análisis de datos}

Las imágenes y videos obtenidos fueron procesados utilizando el software ImageJ. Se calculó el tamańo y velocidad promedio de los espermatozoides. Para los análisis estadísticos se utilizó el software SPSS. 


\section{Aspectos éticos}

Los especímenes son animales de vida libre y no se encuentran en peligro de extinción. Los autores señalan que se cumplieron todos los aspectos éticos nacionales e internacionales.

\section{RESULTADOS}

La superficie de la cabeza de los espermatozoides no fue alterada en el medio control y con $\beta$-cariofileno (Fig. 1). Los valores promedios de 33,8 $\mathrm{um}^{2}$ en el medio control, $32,33 \mathrm{um}^{2}$ en $\beta$-cariofileno sintético (A) y 33,59 $\mathrm{um}^{2}$ en el extracto de $\beta$-cariofileno (B) respectivamente y el nivel de significancia $\mathrm{p}<0.275$ del análisis de varianza, indica la homogeneidad en las muestras

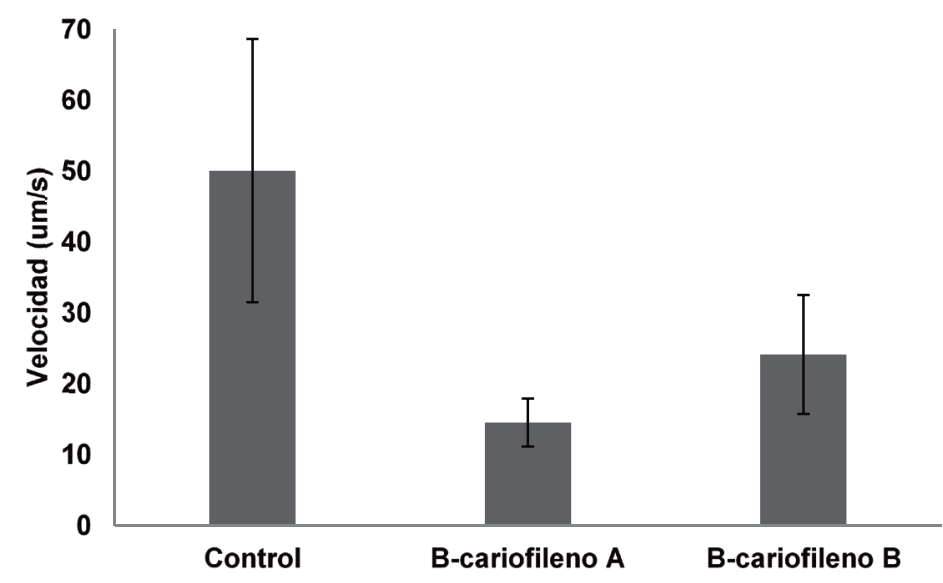

Figura 1. Promedio del área de la cabeza de espermatozoides de Tetrapygus niger en el medio control y en los medios con $\beta$-cariofileno.

El movimiento asimétrico amplio y acelerado del flagelo de los espermatozoides del erizo negro de mar denominado hiperactivación, disminuye en los medios que contienen $\beta$-cariofileno. En la figura 2, se puede observar que en el medio con $\beta$-cariofileno A la hiperactivación disminuye drásticamente (aproximadamente $30 \mathrm{um} / \mathrm{s}$ menos con respecto al control), mientras que en el medio con $\beta$-cariofileno B, la disminución fue del orden de $20 \mathrm{um} / \mathrm{s}$ aproximadamente con respecto al control. Se comprobó mediante el análisis de varianza (prueba de Kruskal Wallis) que existe diferencia significativa entre la hiperactivación en el medio control y los tratamientos con $\beta$-cariofileno (p: 0,00 y 0,02 respectivamente) pero no entre los dos tratamientos con $\beta$-cariofileno (p: 0,10 ).

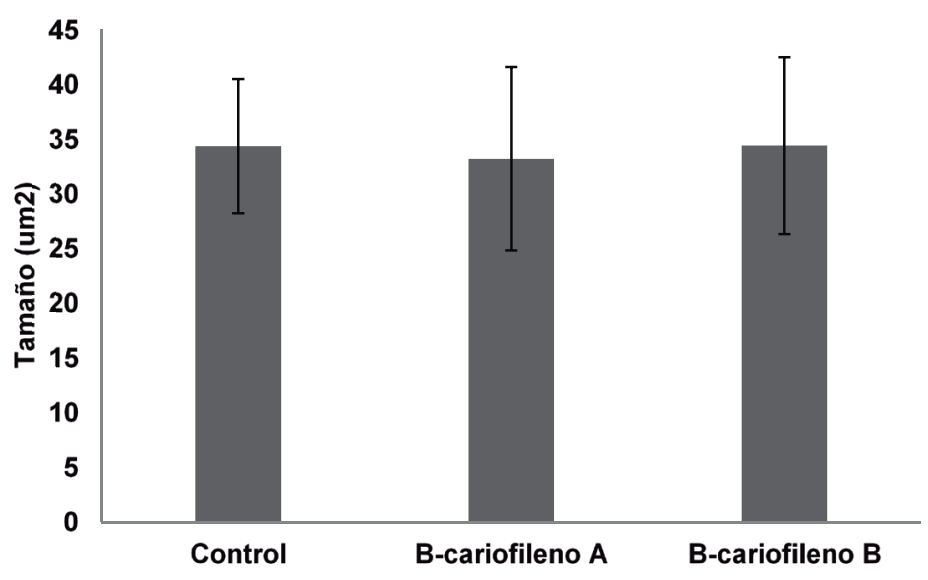

Figura 2. Valores promedios de la velocidad de hiperactivación de los espermatozoides de Tetrapygus niger en los diferentes medios de incubación. 
De la misma manera, el movimiento progresivo y continuo también se alteró en los medios con $\beta$-cariofileno (tabla 1 ). Los espermatozoides que estuvieron en el medio control presentaron progresividad rápida (A) en su totalidad, mientras que el tratamiento con $\beta$-cariofileno $B$ se redujo a $40 \%$. El tratamiento con $\beta$-cariofileno A presentó espermatozoides sólo con movimiento progresivo lento.

Tabla 1. Porcentajes del movimiento progresivo y continuo de los espermatozoides de Tetrapygus niger en los diferentes medios de incubación.

\begin{tabular}{cccc}
\hline \multirow{2}{*}{ Medios de incubación } & \multicolumn{2}{c}{$\begin{array}{c}\text { Porcentaje (\%) de movimiento progresivo y } \\
\text { continuo }\end{array}$} & $\mathrm{C}^{* * *}$ \\
\hline Agua de mar (control) & 100 & 0 & 0 \\
$\beta$-cariofileno A & 0 & 100 & 0 \\
$\beta$-cariofileno B & 40 & 60 & 0 \\
\hline $\mathrm{A}^{*}:$ progresivo rápido: $>$ 25 um/s; $\mathrm{B}^{* *}$ : progresivo lento: entre $5 \mathrm{um} / \mathrm{s} \mathrm{y} 25 \mathrm{um} / \mathrm{s} \mathrm{y}$ \\
$\mathrm{C}^{* * *}:$ no progresivo: $<5 \mathrm{um} / \mathrm{s}$.
\end{tabular}

Con respecto a la fecundación in vitro, esta disminuyó tras el tratamiento de los espermatozoides con $\beta$-cariofileno. El porcentaje de fecundación con los espermatozoides del medio control fue de $100 \%$; por otro lado, los óvulos fecundados con espermatozoides expuestos al extracto de $\beta$-cariofileno B llegaron a 96,76\%, mientras que en el tratamiento con $\beta$-cariofileno A la fecundación se redujo a $79,50 \%$ (tabla 2$)$.

Tabla 2. Porcentaje de óvulos fecundados por espermatozoides de Tetrapygus niger en los diferentes medios de incubación.

\begin{tabular}{ccc}
\hline \multirow{2}{*}{ Medios de incubación } & \multicolumn{2}{c}{ Porcentaje (\%) de fecundación in vitro } \\
& Fecundados & No fecundados \\
\hline Agua de mar (control) & 99,20 & 0,80 \\
$\beta$-cariofileno A & 79,50 & 20,50 \\
$\beta$-cariofileno B & 96,76 & 3,24 \\
\hline
\end{tabular}

\section{DISCUSIÓN}

En este trabajo se presentan los resultados preliminares de la capacidad fecundante del espermatozoide de $T$. niger "erizo negro de mar" en presencia del sesquiterpeno $\beta$-cariofileno obtenido de P. nigrum "pimienta negra" o sintético.

Los espermatozoides de $T$. niger no alteran su organización estructural en presencia de los medios que contienen $\beta$-cariofileno, lo que es muy importante para el inicio de los mecanismos que conducen a la capacidad fecundante (Castellano-Torres et al., 2010). La cabeza, dependiendo de la especie, puede medir entre 2 y 5 \m, contiene un núcleo haploide cuya cromatina está altamente condensada y un acrosoma en la región apical de la cabeza, el cual contiene enzimas hidrolíticas que permiten al espermatozoide atravesar las cubiertas del óvulo (Sardet \& Tiney, 1970), el promedio del área de la cabeza de T. niger fue de $33,8 \mathrm{um}^{2}$.

Gertsch (2008) mostraron que el $\beta$-cariofileno es agonista selectivo del receptor de cannabinoide tipo 2 (CB2). El receptor CB2 activado se asocia a una proteína $\mathrm{G}$ heterotrimérica inhibitoria $\left(\mathrm{G}_{\mathrm{i} / \mathrm{o}}\right)$ pero no a proteínas $G$ estimulantes ( $\left.G_{s}\right)$ (Demuth \& Molleman, 2006). Cuando el receptor de membrana reconoce a su ligando ocurre un cambio de GDP a GTP en la subunidad $\alpha$, la cual pierde afinidad por las otras dos subunidades y se libera del heterotrímero (Childers \& Deadwyler, 1996). La subunidad $\alpha$ ligada a GTP se liga a diversas proteínas, incluyendo adenilciclasas. La unión de la subunidad alfa de $G_{\mathrm{i} / \mathrm{o}}$ a la adenilciclasa inhibe su actividad evitando la formación de cAMP a partir de ATP (Milde et al., 2013). Baja concentración intracelular de cAMP no es capaz de activar la proteína quinasa A (PKA) la cual 
en su forma activa estimula a las quinasas de tirosina espermáticas que conducen una cascada de fosforilación involucrada en la motilidad espermática (Visconti et al., 1995). Estas afirmaciones podrían explicar que el $\beta$-cariofileno promueve la disminución de los valores de hiperactivación en los espermatozoides de erizo negro de mar significativamente.

Por otro lado, las subunidades $\beta / \gamma$ de proteínas $G$ pueden inhibir los canales de calcio mediante su ligamiento a estos (De Waard et al., 1997). Los cannabinoides son capaces de inhibir la abertura de los canales de calcio, probablemente a través del mecanismo anteriormente mencionado (Chang et al., 1993) alterando las concentraciones intracelulares de calcio que evitarían la hiperactivación espermática, la que requiere concentraciones en el rango de 100 300 nanomolar para que ocurra (Pereira et al., 2015). Además, la entrada de calcio al citoplasma espermático es importante para iniciar la reacción del acrosoma (Chang et al., 1993) y se produzca la fecundación del óvulo. Estos mecanismos podrían explicar el efecto en la fertilidad observada tras el tratamiento con $\beta$-cariofileno.

En conclusión, es posible que el $\beta$-cariofileno, al igual que otras sustancias cannabinoides, altere la motilidad espermática y también bloquee la reacción del acrosoma. Sin embargo, ya que no se realizó una medición del grado de pureza del extracto de $\beta$-cariofileno a partir de la pimienta negra, no podemos descartar la posibilidad de que otros compuestos hayan contribuido en la reducción de la motilidad y los porcentajes de fecundación observados. Ensayos con extractos con pureza verificada deben ser realizados para confirmar las evidencias observadas en este estudio.

\section{REFERENCIAS BIBLIOGRÁFICAS}

Agirregoitia, E.; Carracedo, A.; Subirán, N.; Valdivia, A.; Agirregoitia, N.; Peralta, L.; Velasco, G. \& Irazusta. 2010. The CB2 cannabinoid receptor regulates human sperm cell motility. Fertility and Sterility, 93: 1378-1387.

Bernabó, N.; Saponaro, I.; Mattioli, M. \& Barboni, B. 2012. Signaling strategy in spermatozoa activation of sea urchin, C. elegans and human: three different players for the same melody. Journal Bioengineer \& Biomedical Science, S5: 2-6.

Castellano-Torres, L.E.; Martínez-Cadena, G.; LópezGodínez, J.; Cuéllar-Mata, P. \& García-Soto,
J.J. 2010. Los gametos de erizo de mar como modelo de estudio de la fecundación. Revista enlace químico, 7: 14-26.

Chang, M.; Berkery, D.; Schuel, R.; Laychock, S.; Zimmerman, A.; Zimmerman, S. \& Schuel, H. 1993. Evidence for a cannabinoid receptor in sea uchin sperm and its role in blockade of the acrosome reaction, 36: 507-516.

Childers, S. \& Deadwyler, S. 1996. Role of cyclic AMP in the actions of Cannabinoid receptors. Biochemical Pharmacology, 52: 819-827.

De Waard, M.; Liu, H.; Walker, D.; Scott, V.; Gurnett, C. \& Campbell, K. 1997. Direct binding of G-protein $\beta$ complex to voltage-dependent calcium channels. Nature, 385: 446-450.

Demuth, D. \& Molleman, A. 2006. Cannabinoid signaling. Life Sciences, 78: 549-563.

Gertsch, J. 2008. Antiinflammatory cannabinoids in diet - towards a better understanding of CB2 receptor action? Communicative $\&$ Integrative Biology, 1: 26-28.

Jayaprakasha, G.K.; Mohan, J.; Rao, L. \& Sakariah, K.K. 2003. Volatile constituents from Cinnamomum zeylanicum fruit stalks and their antioxidant activities. Journal of Agricultural and Food Chemistry, 51: 4344-4348.

Kamei, N. \& Glabe, Ch.G. 2003. The species-specific egg receptor for sea urchin sperm adhesion is EBR1, a novel ADAMTS protein. Genes \& Development, 17: 2502-2507.

Lu, H.C. \& Mackie, K. 2016. An introduction to the endogenous cannabinoid system. Biology Psychiatry, 79: 516-525.

Mcpartland, J.M.; Agraval, J.; Gleeson, J.; Heasman, K. \& Glass, M. 2006. Cannabinoid receptors in invertebrates. Journal of Evolutionary Biology, 19: 366-373.

Milde, M.; Rinne, A.; Wunder, F.; Engelhardt, S. \& Bunemann, M. 2013. Dynamics of Gßi1 interaction with type 5 adenylate cyclase reveal the molecular basis for high sensitivity of Gimediated inhibition of cAMP production. Biochemistry Journal, 454: 515-523 
Mockute, D.; Bernotiene, G. \& Judzentiene, A. 2001. The essential oil of Origanum vulgare L. ssp. vulgare growing wild in vilnius district (Lithuania). Phytochemistry, 57: 65-69

Neil, A.T. \& Vacquier, V.D. 2004. Ligands and receptors mediating signal transduction in sea urchin spermatozoa. Reproduction, 127: 141-149.

Olaechea, P.; Paez, J.J. \& Gonzales-Figueroa, H. 2006. Desarrollo embrionario de Tetrapygus niger (Molina, 1782) "erizo negro" en diferentes temperaturas. Biotempo, 6: 27-31.

Orav, A.; Stulova, I.; Kailas, T. \& Müürisepp, M. 2004. Effect of storage on the essential oilcomposition of Piper nigrum L. fruits of different ripening states. Journal of Agricultural and Food Chemistry, 52: 2582-2586.

Pereira, R.; Sá, R.; Barros, A. \& Sousa, M. 2015. Major regulatory mechanisms involved in sperm motility. Asian Journal of Andrology, 19: 5-14.

Sarde, T.D. \& Tilney, L. 1970. Origin of acrosomal process is actin complex with membrane precursor? Cell Biology Repoduction, 1: 193200.

Schuel, H.; Chang, M.; Berker, C.; Schuel, D.; Zimmerman, R. \& Zimmerman, S. 1991. Cannabinoids inhibit fertilization in sea urchins by reducing the fertilizing capacity of sperm. Pharmacology, Biochemistry and Behavior, 40: 609-615.

Visconti, P.; Moore, G.; Bailey, J.; Leclerc, P.; Connors, S.; Pan, D.; Olds-Clarke, P. \& Kopf, G. 1995. Capacitation of mouse spermatozoa. II. Protein tyrosine phosphorylation and capacitation are regulated by a cAMP-dependent pathway. Development, 121: 1139-1150.

Zhou, B.; Tan, M.; Lu, J.F.; Zhao, J.; Xie, A.F. \& Li, S.P. 2012. Simultaneous determination of five active compounds in Chimonanthus nitens by double-development HPTLC and scanning densitometry. Chemistry Central Journal, 6: 46.

Received November 7, 2019.

Accepted November 19, 2019. 